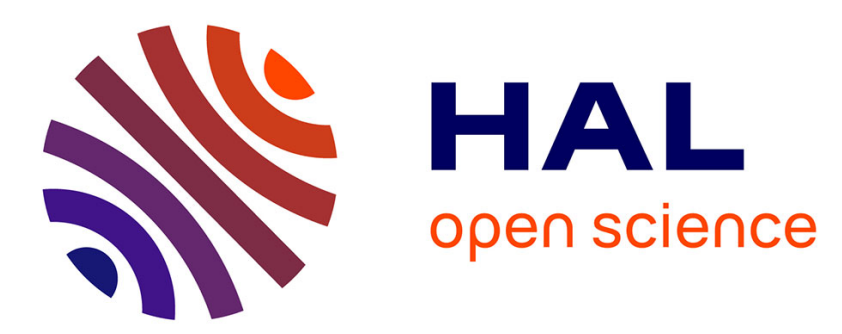

\title{
High-Resolution Shortwave Infrared Imaging of Vascular Disorders Using Gold Nanoclusters
}

Zhixi Yu, Benjamin Musnier, Maxime Henry, K David Wegner, Benoit Chovelon, Agnes Desroches-Castan, Arnold Fertin, Ute Resch-Genger, Sabine Bailly, Jean-Luc Coll, et al.

\section{To cite this version:}

Zhixi Yu, Benjamin Musnier, Maxime Henry, K David Wegner, Benoit Chovelon, et al.. HighResolution Shortwave Infrared Imaging of Vascular Disorders Using Gold Nanoclusters. ACS Nano, 2020, 10.1021/acsnano.0c01174 . hal-02945280

\author{
HAL Id: hal-02945280 \\ https://hal.science/hal-02945280
}

Submitted on 28 Sep 2020

HAL is a multi-disciplinary open access archive for the deposit and dissemination of scientific research documents, whether they are published or not. The documents may come from teaching and research institutions in France or abroad, or from public or private research centers.
L'archive ouverte pluridisciplinaire HAL, est destinée au dépôt et à la diffusion de documents scientifiques de niveau recherche, publiés ou non, émanant des établissements d'enseignement et de recherche français ou étrangers, des laboratoires publics ou privés. 


\section{High-resolution of Vascular Disorders by Shortwave Infrared Imaging using Gold Nanoclusters}

Zhixi Yu ${ }^{l}$, Benjamin Musnier ${ }^{l}$, Maxime Henry ${ }^{l}$, K. David Wegner ${ }^{2}$, Benoit Chovelon ${ }^{3}$, Agnès Desroches-Castan ${ }^{4}$, Arnold Fertin ${ }^{5}$, Ute Resch-Genger ${ }^{2}$, Sabine Bailly, Jean-luc Coll ${ }^{*}$, Yves Usson $^{5}$, Véronique Josserand ${ }^{1}$, Xavier Le Guével ${ }^{*}$

${ }^{1}$ Cancer Targets \& Experimental Therapeutics, Institute for Advanced Biosciences (IAB), University of Grenoble Alpes (UGA)/ INSERM-U1209 / CNRS-UMR 5309- Grenoble, France

${ }^{2}$ BAM Federal Institute for Materials Research and Testing, Richard-Willstaetter-Str. 11, 12489 Berlin, Germany.

${ }^{3}$ Institut de Biologie et Pathologie, CHU de Grenoble-Alpes, France

${ }^{4}$ Biology of Cancer and Infection Laboratory, University Grenoble Alpes, Inserm, CEA,38000 Grenoble, France

${ }^{5}$ TIMC-IMAG, University of Grenoble Alpes, CNRS-UMR 5525, Grenoble, France

ABSTRACT: We synthesized a generation of water-soluble, atomically precise gold nanoclusters (Au NCs) with anisotropic surface containing short dithiol pegylated chain. These $\mathrm{Au}$ NCs exhibit a high brightness (QY 6\%) in the shortwave infrared (SWIR) spectrum with a detection above $1250 \mathrm{~nm}$ and showed a slow elimination from blood with a weak accumulation in organs. We also developed a non-invasive, whole-body vascular imaging system in the SWIR window with high-resolution, benefiting from a series of Monte Carlo image processing of the images. The imaging process enabled to improve contrast by one order of magnitude and enhance by $54 \%$ the spatial resolution. After systemic administration of these nanoprobes in mice, we could quantify vessel complexity in depth ( $>4 \mathrm{~mm})$. Using Bmp9 deficient mice, we can detect very subtle vascular disorders noninvasively. The combination of these anisotropic surface charged gold nanoclusters plus an improved SWIR imaging device allows then a precise mapping at high resolution and in depth of the organization of the vascular network in live animals. 
KEYWORDS: shortwave infrared fluorescence, gold nanoclusters, vascular disorder, monte carlo restoration imaging processing.

In vivo infrared imaging has experienced major breakthroughs over the past few years with potential applications in cancer and cardio-vascular diagnostics ${ }^{1}$. Hongjie Dai's team was among the first who developed emitters for the shortwave infrared region (SWIR, 900-1700 $\mathrm{nm}$ ), also called NIR II. Due to the weak photon absorption, the low autofluorescence, and reduced scattering by tissues at these wavelengths compared to NIR I (700-900 nm) and the visible region, they were able to reach a high spatial and temporal resolution through a few millimeters of tissue sufficient to image brain blood circulation through the intact skull ${ }^{2}$ and in ischemic femoral arteries ${ }^{3}$. Using SWIR imaging, Bawendi et al. recently evaluated the metabolic turnover rates of lipoproteins in several organs in real-time as well as the heartbeat and breathing rates in awake and unrestrained animals ${ }^{4}$. Based on these data, they generated a detailed three-dimensional quantitative flow map of the mouse brain vasculature, which demonstrates the high potential of SWIR-imaging applications.

However, only few SWIR emitting contrast agents are available that possess high quantum yields (QYs), good biocompatibility and low accumulation in organs. Although in vivo imaging studies have been performed using SWIR-emitting quantum dots (QDs) ${ }^{4}$, the downshifting emission of lanthanide-based nanomaterials ${ }^{5}$, and new organic donor-acceptordonor (D-A-D) type organic fluorophores ${ }^{6}$, these materials still have some drawbacks. This includes for example, their possible toxicity related to the toxicity of their constituents in the case of most QDs and low QYs $<1 \%$ for the organic molecules. A relatively new class of NIR II emitters are ultra-small gold nanoclusters $(\mathrm{Au} \mathrm{NCs})^{7}$. Recent studies with Au NCs stabilized by zwitterionic sulfobetaine ligands ( $\mathrm{AuZw}$ ) showing a reasonably high QY (3.8\%), enabled to detect blood vessels using photoluminescence (PL) and revealed efficient renal clearance $^{8}$ 
In this work, we developed water-soluble Au NCs stabilized by co-ligands with emission in the SWIR, which exhibit a high brightness, a high photostability, long blood circulation times, and a low toxicity. We demonstrate the ability of this promising new contrast agent to image the vascular network of mice with vascular disorders in the second window of the SWIR spectrum (1250-1700 nm) with one-order magnitude contrast enhancement and 54\% increase in spatial resolution enabled by the use of Monte Carlo image processing. The non-invasive imaging processing, segmentation and analyzes were validated in transgenic mice inactivated for the Growth factor $B m p 9$ that were previously described with vascular disorders ${ }^{9,10}$ based on the measurement of the fractal dimension of vessels.

\section{RESULTS \& DISCUSSION}

The here developed Au NCs, named AuMHA/TDT, were prepared by a wet chemistry route using mercaptohexanoic acid (MHA) and tetra(ethyleneglycol) dithiol (TDT) as co-ligands with the molar ratio Au: ligand $=1: 4$ and MHA: TDT $=1: 3$ (Figure 1a, see experimental details). Mass spectrometry revealed a high monodispersity of an $11 \mathrm{kDa}$ species (Figure S1). The average size of the semi-crystalline metal core was determined by high resolution transmission electron microscopy (HR-TEM) to $2.1 \pm 0.6 \mathrm{~nm}$ (Figures 1b, S2) and the hydrodynamic diameter of AuMHA/TDT in water to $1.90 \pm 0.02 \mathrm{~nm}$ as derived from DOSYNMR (Figure S3). The water-soluble AuMHA/TDT NCs have a negative surface charge at $\mathrm{pH} 7$ of around $\xi=-20 \mathrm{mV}$, which renders them very stable over the long period of time. The addition of the short dithiol molecules TDT on the Au NC surface results in a striking modification of the optical properties, as recently reported by us for another type of anisotropic Au NCs prepared with hexa(ethylene glycol) dithiol as co-ligand ${ }^{11}$. Indeed, the incorporation of TDT leads to the presence of new NIR absorbance features at $800 \mathrm{~nm}, 910$ $\mathrm{nm}$ and $1140 \mathrm{~nm}$ (Figure 1c). The anisotropic surface of Au NCs also influences drastically 
the PL signal in the SWIR region with a 9-fold increase of PL intensity in water accompanied by PL emission at longer wavelengths (Figure 1d). The spectral position and shape of the emission is independent of the excitation wavelength (Figure S4) and the average lifetime derived from the multiexponential decay detected at $\lambda_{\mathrm{em} .}=930 \pm 20 \mathrm{~nm}$ is $\left\langle\tau_{\text {int. }}>=449.0 \mathrm{~ns}\right.$ (Figure S5). We compared the PL of AuMHA/TDT to the emission of other water-soluble Au NCs synthesized in our laboratories, i.e., $\mathrm{AuMHA}^{11}, \mathrm{AuZw}^{12}$, and $\mathrm{Au}_{25} \mathrm{SG}_{18}{ }^{13}$ in different spectral windows in the SWIR. The images in Figure 1e show an up to 12-fold more intense signal for AuMHA/TDT using long pass filters above $1250 \mathrm{~nm}$ confirming the superior brightness of our new Au NCs in water as compared to the existing SWIR-emissive ones. We estimated then a quantum yield at $\sim 6 \%$, in the same range as Au NCs stabilized by both ligands mercaptohexanoic acid (MHA) and dithiol hexa(ethyleneglycol) that we previously prepared ${ }^{11}$. This QY is higher than the others $\mathrm{Au}$ NCs already in the literature for $\mathrm{Au}_{25} \mathrm{SG}_{18}$ $(0.67 \%)^{14}$, AuMHA $(0.9 \%)^{11}$, and AuZw $(3.8 \%)^{8}$. The PL features of AuMHA/TDT remain unchanged in water and in the presence of 10\% serum (Figure S6). Illumination studies in water, with $\mathrm{NaCl}(0.9 \%)$, and in the presence of bovine serum albumin (BSA; $50 \mathrm{mg} / \mathrm{mL}$ ) at different incubation times demonstrate the high photostability of these emitters (Figure S7). Surprisingly, in the presence of blood, the PL increases over time. A similar behavior has also been reported for $\mathrm{ICG}^{15}$ and is tentatively ascribed to specific interactions with proteins/complement components or uptake by red blood cells.

Cytotoxicity experiments (Figure S8) have been conducted using MTT assays with a human embryonic cell line (HEK 293) and human (A549) or murine (4T1) cancer cell lines in the presence of increasing concentrations of AuMHA/TDT up to $45 \mu \mathrm{M}(\sim 500 \mu \mathrm{g} / \mathrm{mL})$. The absence of significant cell death suggests the low toxicity of these contrast agents and allowed us to move forward to in vivo studies. 
The half-life of Au NCs in mice is highly dependent on the hydrodynamic diameter of the $\mathrm{NCs}^{12,16}$ and their density ${ }^{17}$. For instance, small $\mathrm{Au}_{25} \mathrm{SG}_{18} \mathrm{NCs}$ show an extremely fast clearance with a half-life of less than 2 min as compared to 6 min for the larger AuZw ${ }^{12}$. By elemental analysis (ICP-MS) after AuMHA/TDT administration, we measured a half-life of $\mathrm{t}_{1 / 2 \mathrm{a}}=0.43 \pm 0.05 \mathrm{~h}$ and a half-elimination of $\mathrm{t}_{1 / 2 \beta}=19.54 \pm 0.05 \mathrm{~h}$ in nude mice (Figure 2a). These two values are more than 5 times longer than those obtained with the previous applied $\mathrm{Au}$ NCs. This prolonged blood circulation time enables the detection of SWIR signals from AuMHA/TDT in the blood vessels in mice up to 30 minutes after injection.

In vivo fluorescence measurements also show the renal clearance of these ultra-small particles with signal in the bladder at $3 \mathrm{~h}$ post injection and with an elimination from the liver observed between $5 \mathrm{~h}$ to $24 \mathrm{~h}$, respectively (Figure $2 \mathbf{b}$ ).

One of the major advantages of the SWIR spectral window is the low autofluorescence, which enables to reduce the amount of the contrast agent necessary to achieve a high signal to noise ratio. In our case, we could detect AuMHA/TDT NCs in in vitro samples at concentrations as low as $80 \mathrm{nM}$ with a signal-to-noise ratio of 3.4 (Figure S9). This enabled us to follow the biodistribution of AuMHA/TDT in mice by fluorescence imaging after $5 \mathrm{~h}$ or $24 \mathrm{~h}$ postinjection.

Results from ex vivo fluorescence measurements in different organs (Figure 2c), confirmed by ICP-MS measurements (Figure S10), suggest a renal elimination of the Au NCs and an accumulation of the Au NCs mainly in the kidney, the liver, and the spleen with PL signal dropping by $40 \%$ between $5 \mathrm{~h}$ and $24 \mathrm{~h}$, which suggests an elimination or a metabolization of AuMHA/TDT over time.

Non-invasive real-time SWIR imaging was performed after intravenous injection of AuMHA/TDT $(200 \mu \mathrm{L} ; 360 \mu \mathrm{M})$ in vivo in 129/Ola mice using a $830 \mathrm{~nm}$ laser as light excitation source $\left(50 \mathrm{~mW} / \mathrm{cm}^{2}\right)$ and a long-pass filter LP1250 $\mathrm{nm}$ in the emission channel. 
Under these conditions, the AuMHA/TDT NCs provided a clear and outstanding visualization of the vascular network as shown in movie 1.

As expected, there is a clear improvement of the spatial resolution in the SWIR is strongly improved as compared to the NIR I (Figure 3a). We performed post-image processing with the Monte Carlo Constrained Restoration (MCR) method to further improve the spatial resolution in depth and to overcome the scattering from the skin and the tissues. Image processing greatly enhances the spatial resolution as seen in Figure $\mathbf{3 b}$. Comparing the quality of the images before and after MCR using a quantitative measure of the contrast (Figure 3c) demonstrates an improvement in contrast by one order of magnitude, highlighting the potential of this restoration. The determination of the transverse section of blood vessels from the SWIR images of a mouse leg depicted in Figure 3d highlights the excellent resolution after MCR processing and the ability to obtain highly detailed in vivo imaging of the whole-body blood vasculature and of the blood brain vasculature (Figure S11).

Image processing was then pushed one step further using a high-pass filter to reduce scattering deeper under the skin. Restorations of the first movie by MCR plus the high pass filter underline the striking improvement of the resolution (movie 2, Figure S12) allowing the visualization of the vascular network at more than $4 \mathrm{~mm}$ penetration depth (Figure 4a). We could also easily track the contrast agent (SWIR-emitting AuMHA/TDT) in mice especially in the first seconds after injection (movie 3, Figure 4b).

To demonstrate the potential of combining SWIR imaging, image restoration, and image analysis for biomedical applications, non-invasive vascular imaging was performed in mice previously described with vascular disorders due to the inactivation of the gene encoding $B m p 9^{9,10}$ and compared to wild-type mice (WT). Bmp9 has been identified as a high affinity ligand for the endothelial specific receptor ALK1 (activin receptor-like kinase 1), that is mutated in the rare vascular disease HHT (hereditary Haemorrhagic Telangiectasia) ${ }^{18}$. We 
have recently shown that Bmp9 deletion in the 129/Ola genetic background results in dilated liver vessels that ultimately lead to liver fibrosis ${ }^{10}$.

Bmp9-KO mice and WT mice were placed in supine position and were injected intravenously with AuMHA/TDT for real-time SWIR imaging from $\mathrm{t}=0$ to $\mathrm{t}=15 \mathrm{~min}$. MCR was performed on raw images followed by Frangi's filtering and segmentation (see methods) as illustrated in Figure 5a. Then, analyses of the segmentation, which provides blood network mapping were plotted considering the length of the vessels or the number of vessel "branches" (segments delimited by forks or crossings) as a function of the fractal dimension (Figure 5b). Fractal dimensional is referred here as the level of complexity of vessels with a numerical value between 1 (straight line tube) to 2 (highly swaying vessel in 2-dimensional section). Analyses show a clear distinction in skin vessels between Bmp9-KO and WT mice considering both vessel lengths and branches. The significant $4 \%$ increase of the fractal dimension for Bmp9KO compared to WT mice, which is correlated to the vessel distortion, confirms then the hypothesis of tortuous vessels due to a defect in vessel maturation ${ }^{10}$. Thus, this non-invasive method allowed us for the first time to detect a vascular disorder present in the $B m p 9-\mathrm{KO}$ mice with such a high level of precision.

\section{CONCLUSION}

In conclusion, we report here the design of a new bright SWIR-emitting contrast agent with prolonged circulation and efficient elimination that enables the visualization of the vascular network with enhanced-spatial resolution in depth using a series of MCR processing steps. Both image processing and segmentation analyses enabled to distinguish non-invasively vascular disorders in mice with good confidence. To the best of our knowledge, this is the first time that such results were obtained non-invasively in depth and in real time using whole-body optical imaging. This study also highlights the considerable advantages of 
combined research on new contrast agents and on image processing and analyses to improve the sensitivity of SWIR imaging for advanced biomedical applications.

\section{MATERIALS \& METHODS}

Matrix Assisted Laser Desorption Ionisation - Time of Flight (MALDI-TOF). Au NCs sample was diluted in the matrix CHCA (Alpha-Cyano-4-Hydroxy-3-Iodocinnamic Acid) with $0.1 \%$ trifluoroacetic acid (TFA) in a mixture water/acetonitrile 50/50 v/v. Measurements were performed in positive linear mode on an Autoflex speed instrument from Brucker.

Nuclear magnetic resonance spectroscopy (NMR) experiments were carried out at $298 \mathrm{~K}$ with a Bruker AVANCE III $500 \mathrm{MHz}$ spectrometer equipped with a cryo-probe Prodigy. For each sample the concentration was $\sim 2 \mathrm{mM}$ in $\mathrm{D}_{2} \mathrm{O}$ and $\mathrm{pH}$ 7. Diffusion ordered NMR spectroscopy (DOSY) experiments were run using the standard "ledbpg2s" Bruker sequence with linear gradient stepped between $2 \%$ and $98 \% .32$ scans were recorded for each gradient step. Data processing were performed using the maximum entropy algorithm from Dynamics Center, a Bruker's NMR software to obtain the diffusion coefficient D. An average value of D was used for the hydrodynamic diameter (HD) calculation according to the Stokes-Einstein equation which assumes that molecules are spherical:

$$
\mathrm{HD}=\mathrm{kBT} / 3 \mathrm{D} \pi \eta
$$

where $\mathrm{kB}$ is the Boltzmann constant, $\mathrm{T}$ is the temperature, $\eta$ is the viscosity of the solvent $\left(\eta \mathrm{D}_{2} \mathrm{O}=1.232\right.$ 10-3 Pa.s at 298K). Standard 1D and 2D (NOESY, COSY, TOCSY and HSQC) spectra were recorded using standard presaturation on the water signal.

Zeta potential of $\mathrm{Au}$ NCs dispersed in water or in PBS buffer with $10 \%$ serum were measured with a Zetasizer from Malvern instruments.

Metal core sizes were determined by high resolution transmission electron microscopy with an $200 \mathrm{kV}$ monochromated TEM using dispersed Au NCs on ultra-fine carbon films. 
Absorption spectra of diluted AuNC samples were recorded on a UV-vis-NIR spectrophotometer Cary5000 between 350 and $1700 \mathrm{~nm}$.

Steady-state photoluminescence spectra were measured from $600-1750 \mathrm{~nm}$ with a calibrated FSP 920 (Edinburgh Instruments, Edinburgh, United Kingdom) spectrofluorometer equipped with a nitrogen-cooled PMT R5509P.

Time-resolved measurements were performed in the wavelength region of $930 \pm 20 \mathrm{~nm}$ using a FLS 920 (Edinburgh Instruments, Edinburgh, United Kingdom) lifetime spectrofluorometer equipped with an EPL-510 (Edinburgh Instruments, Edinburgh, United Kingdom) picosecond pulsed diode laser (excitation wavelength of $510 \pm 10 \mathrm{~nm}$; power of $5 \mathrm{~mW}$ ) and a fast PMT R2658P from Hamamatsu, respectively.

Relative measurements of photoluminescence QYs $(\Phi f, \mathrm{x})$ were performed using the dye IR125 dissolved in dimethylsulfoxide (DMSO as reference. The QY of this dye was previously determined absolutely to $\Phi f, \mathrm{st}=0.23$ ). The relative QY were calculated according to the formula of Demas and Crosby6, see equation below.

$$
\Phi_{f, x}=\Phi_{f, s t} \frac{F_{x}}{F_{s t}} \cdot \frac{f_{s t}\left(\lambda_{e x, s t}\right)}{f_{x}\left(\lambda_{e x, x}\right)} \cdot \frac{n_{x}^{2}\left(\lambda_{e x, x}\right)}{n_{s t}^{2}\left(\lambda_{e x, s t}\right)}
$$

The subscripts $x$, st, and ex denote sample, standard, and excitation respectively. $f\left(\lambda_{\text {exc. }}\right)$ is the absorption factor, $\mathrm{F}$ the integrated spectral fluorescence photon flux, and $\mathrm{n}$ the refractive index of the solvents used (DMSO for IR125; water for Au NCs).

All spectroscopic measurements were done in a $1 \mathrm{~cm}$ quartz cuvettes from Hellma $\mathrm{GmbH}$ at room temperature using air-saturated solutions.

Cytotoxicity experiments. HEK, 4T1, A549 cells were seeded in a 96-well plate (5 000 cells/well in $100 \mu \mathrm{L}$ of DMEMc) 1 day before the experiment. Au NCs were diluted in DMEMc to reach the following final concentrations: $25,100,250$, and $500 \mu \mathrm{gA} / \mathrm{mL}$ and added to the cells. Cell viability was evaluated $24 \mathrm{~h}$ after Au NCs incubations in triplicate. For positive and negative controls, cells with or without $1 \mu \mathrm{M}$ staurosporine treatment (cell-death 
inducer) were also prepared. In order to verify the absence of artefact due to the absorption of the Au NCs, wells with only Au NCs in DMEMc were also prepared. Then $20 \mu \mathrm{L}$ of MTS reagent (Cell-Titer 96, Promega) was added to each well and further incubated at $37{ }^{\circ} \mathrm{C}$ for 2 h. The cell density was then determined by the absorbance at $486 \mathrm{~nm}$ using a microplate spectrophotometer (Fluostar Omega BM6 LABTECH). The percentages of cell viability were compared using the non-parametric Kruskal-Wallis test. All analyses were performed using GraphPad Prism software. Statistical significance was defined as p values $<0.001$.

NIR I imaging was performed with a NIR 2D-Fluorescence Reflectance Imaging device (Fluobeam $800^{\circ}$, Fluoptics, France). The excitation is provided by a class 1 expanded laser source at $780 \mathrm{~nm}$ and the irradiance on the imaging field is $10 \mathrm{~mW} / \mathrm{cm}^{2}$. The fluorescence signal is collected by a CCD through a High pass filter with a high transmittance for wavelength $>830 \mathrm{~nm}$.

SWIR imaging was performed using a Princeton camera 640ST (900-1700 nm) coupled with a laser excitation source at $\lambda=830 \mathrm{~nm}\left(50 \mathrm{~mW} / \mathrm{cm}^{2}\right)$. We use short-pass excitation filter at $1000 \mathrm{~nm}$ (Thorlabs) and long-pass filters on the SWIR camera from Semrock (LP1064 nm, LP1319 nm) and Thorlabs (LP1250 nm, LP1300 nm, LP1500 nm). $25 \mathrm{~mm}$ or $50 \mathrm{~mm}$ lenses with 1.4 aperture (Navitar) were used to focus on the samples or mice.

Tubes containing AuNC solution and $10 \mu \mathrm{L}$ drops of each samples were placed in front of the camera using the $50 \mathrm{~mm}$ lens and various long pass filters (LP1064 nm, LP1250 nm, LP 1300 nm, LP1319 nm, LP1500 nm). Analyses were performed using FIJI software.

Mice were imaged after intravenous injection $(200 \mu \mathrm{L}$ of $\mathrm{Au} \mathrm{NCs}$ at $360 \mu \mathrm{M})$ using the 25 $\mathrm{mm}$ or $50 \mathrm{~mm}$ lenses and LP1250 $\mathrm{nm}$ at different exposure times (25ms to $2 \mathrm{~s}$ ). Ex vivo fluorescence imaging on isolated organs and plasma samples were performed using the 50 mm lens and LP1064 nm. 
Inductively coupled plasma-mass spectrometry (ICP-MS) was performed to determine $\mathrm{Au}$ content in organs and in plasma samples at different time point using a Thermo X serie II, spectrometer (Thermo Electron, Bremen, Germany), which was equipped with an impact bead spray chamber and a standard nebulizer $\left(1 \mathrm{~mL} \cdot \mathrm{min}^{-1}\right)$. For sample preparation, the organs and plasma samples were weighted before addition of nitric acid (final concentration 1\%) and $\mathrm{Au}$ content was determined using an external linear calibration curve (between 10 and $100 \mu \mathrm{g} / \mathrm{L}$ of $\mathrm{Au}(\mathrm{III}))$. Indium was used as the internal standard. Determinations were carried out in triplicate.

In vivo experiments. For the biodistribution study, six weeks old NMRI female nude mice (Janvier, France) were anesthetized (air/isoflurane 4\% for induction and 1.5\% thereafter) and were injected intravenously via the tail vein with $200 \mu \mathrm{L}$ of Au NCs at $360 \mu \mathrm{M}$. In vivo SWIR fluorescence imaging was performed before and 5 and 24 hours after injection. Mice were euthanized at 5 or 24 hours post injection ( $n=3$ mice per time point) and organs were harvested for ex vivo fluorescence imaging and ICP measurements.

For the pharmacokinetic study, three other mice were injected and blood samples were collected before and 1, 5, 15, 30, 60, 180, 300, and 1440 min after injection and were centrifuged (10 min at $2000 \mathrm{~g}$ ) to separate plasma. Plasma pharmacokinetic was obtained from fluorescence imaging and ICP-MS measurements after analyses through a noncompartment model (GraphPad Prism 7.00, GraphPad Software, La Jolla California USA). Noninvasive SWIR imaging on mice with vascular disorders. Generation of Bmp9-KO mice in the 129-P2/Ola-Hsd genetic background (named here for easiness 129/Ola) has been previously described ${ }^{9,10}$. Briefly, Bmp9-KO mice in the C57BL/6 genetic background were obtained from Dr Se-Jin Lee (Johns Hopkins University, Baltimore, MD) and back-crossed for 10 generations with 129-P2/Ola-Hsd wild-type mice (Harlan France, Gannat, France). 
All animals experiments followed the institutional guidelines formulated by the European Community for the Use of Experimental Animals were approved by ethics committees (CEA ethic committee for animal breeding and Cometh38 for in vivo imaging) and the French Ministry of Research and Education. (agreement APAFIS\#9436-2017032916298306 and APAFIS\#21916-2019082710189095_v4).

Image restoration of SWIR fluorescent images. SWIR already provides stunning fluorescent images in vivo, however these images still suffer from light diffusion by the tissues and the quality of such images can still be improved using image restoration techniques. We used a Monte Carlo constrained reconstruction (MCR) algorithm based on an original idea by Frieden et al. ${ }^{19}$ for restoring binary images then extended to fluorescent image deconvolution by Colicchio et $a .^{20}$. This algorithm has the advantage of perfectly preserving the amount of information of the image (intensity integral) and achieve powerful contrast and resolution enhancements, while minimizing the ringing artefacts usually encountered with regular iterative constrained deconvolution. However, Monte Carlo algorithms are very computer intensive and parallelisation schemes must be used to speed up calculations.

Assessment of image enhancement. The enhancement of fluorescent images was both assessed by visual inspection (qualitative assessment) and by using a quantitative measurement. This was achieved by quantifying the contrast $C_{d}$ within the image at different neighbourhoods (distance $d=1,2$ and 4 pixels). The contrast $C_{d}$ was expressed as the gradient integral of the image.

$$
C_{d}=\frac{\sum_{1}^{r-d} \sum_{1}^{c-d}\left({\frac{\delta i}{\delta_{d} x}}^{2}+{\frac{\delta i}{\delta_{d} y}}^{2}\right)}{\langle i\rangle^{2}}
$$

with

$$
\frac{\delta i}{\delta_{d} x}=i(x+d, y)-i(x, y) ; \quad \frac{\delta i}{\delta_{d} y}=i(x, y+d)-i(x, y) ;\langle i\rangle=\frac{\sum_{1}^{r} \sum_{1}^{c} i(x, y)}{r \cdot c}
$$


where $i(x, y)$ is the intensity at pixel $(x, y) ; d$ is the extent of the partial derivate (in pixels) ; $\delta i / \delta_{d} x$ the partial derivate of intensity along $x$ and $\delta i / \delta_{d} y$ the partial derivate of intensity along $y$ at pixel $(x, y) ; r$ is the number of rows and $c$ the number of columns in the image.

Vessel detection and analysis. The analysis of the vascularization was performed by image analysis of the restored images. We used a classical Frangi's filter that was designed to enhance tubular structures in a grey level image ${ }^{21}$. The advantage of Frangi's filter is a good immunity to noise that reduces over-detection. Frangi's filter provides a "tubeness" or "vesselness" probability image to which a probability threshold can be applied in order to obtain a binary mask of the vascular network. After, iterative thinning of the binary mask, a skeleton is obtained and a neighbourhood analysis is applied in order to extract branches, forks and crossings. The statistical analysis of the various features extracted from the skeleton such as: the fractal dimension, total length, the number of branches, the number of forks \& crossings; is used to quantify possible differences between mouse strains.

Synthesis of Au NCs. Chemical products were purchased in Sigma-Aldrich (France) and deionized water was used for all synthesis.

We slightly modified a protocol described by Musnier et al. ${ }^{11}$ to produce the SWIR-emitted $\mathrm{Au}$ NCs using the initial molar ratio Au:Ligand $=1: 4$. Briefly $250 \mu \mathrm{L}$ of $\mathrm{HAuCl}_{4} \cdot 3 \mathrm{H}_{2} \mathrm{O}(20$ $\mathrm{mM}$ ) was added to $4.8 \mathrm{~mL}$ water followed by $4 \mathrm{~mL}$ of the thiolated ligand mixture mercaptohexanoic acid (MHA, $5 \mathrm{mM})$ ) / tetra(ethyleneglycol) dithiol (TDT, $5 \mathrm{mM}$ ) changing colour from yellowish to slightly pale cloudy with a volume ratio $\mathrm{MHA} / \mathrm{TDT}=3 \mathrm{~mL} / 1 \mathrm{~mL}$. After $5 \mathrm{~min}, 250 \mu \mathrm{L}$ of $\mathrm{NaOH}(1 \mathrm{M})$ was added dropwise leading to almost colourless sols. After $5 \mathrm{~min}, 150 \mu \mathrm{L} \mathrm{NaBH}_{4}(20 \mathrm{mM}$ in $0.2 \mathrm{M} \mathrm{NaOH})$ was introduced dropwise under mild stirring and kept under stirring at $350 \mathrm{rpm}$ for 8 hours. Purification of the AuMHA/TDT on 3 
$\mathrm{kDa}$ cut-off filter column (Amicon) were repeated 3 times to stop the reaction and sols were kept stored in the fridge before characterization.

The $\mathrm{Au} \mathrm{NCs}, \mathrm{Au} \mathrm{MHA}^{11}, \mathrm{AuZwMe}_{2}{ }^{8}$ and $\mathrm{Au}_{25} \mathrm{SG}_{18}{ }^{22}$ were synthesized following the protocols described in the literature.

\section{ASSOCIATED CONTENTS}

Supporting Information contains: physico-chemical and photophysical of $\mathrm{Au}$ NCs; cytotoxicity and additional in vivo experiments in mice are available from American Chemical Society or from the author.

\section{AUTHOR INFORMATION}

\section{Corresponding author}

*xavier.le-guevel@univ-grenoble-alpes.fr 0000-0003-3634-7762

*jean-luc.coll@univ-grenoble-alpes.fr 0000-0002-2453-3552

\section{ACKNOWLEDGEMENTS}

XLG would like to thank Ines Häusler for the electron microscopy images (The TEM images were carried out as part of the DFG core facility project "Berlin Electron Microscopy Network (Berlin EM Network)") and Muriel Jourdan for the NMR measurements.

XLG would like to thank Cancéropôle Lyon Auvergne Rhône-Alpes (CLARA), Plan Cancer (C18038CS) and ARC (R17157CC) for their financial support. KDW acknowledges the European Union's Horizon 2020 research and innovation programme under the Marie Sklodowska-Curie grant agreement No.846764. This research was funded by the Institut National de la Santé et de la Recherche Médicale (INSERM, U1036), the Commissariat à 
1'Energie Atomique et aux Energies Alternatives (CEA, DRF/IRIG), the University Grenoble Alpes (UGA, BCI), the Fondation pour la Recherche Médicale (FRM).

There is no conflict of interest.

All applicable international, national, and/or institutional guidelines for the care and use of animals were followed.

a
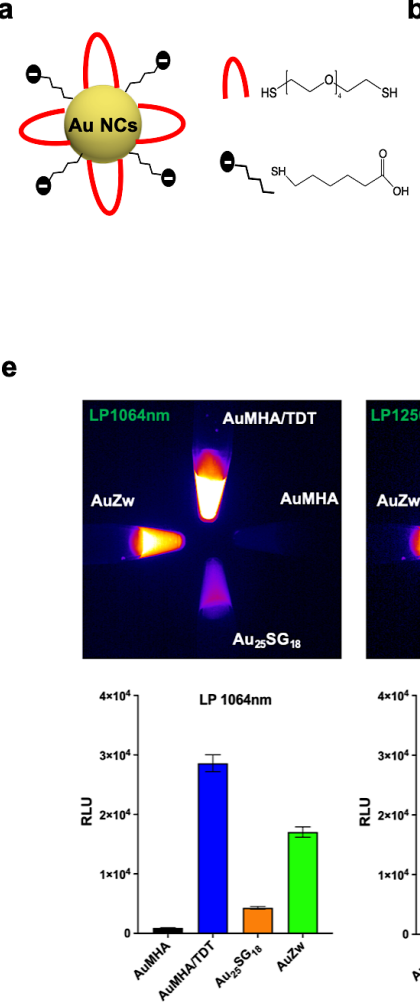

b
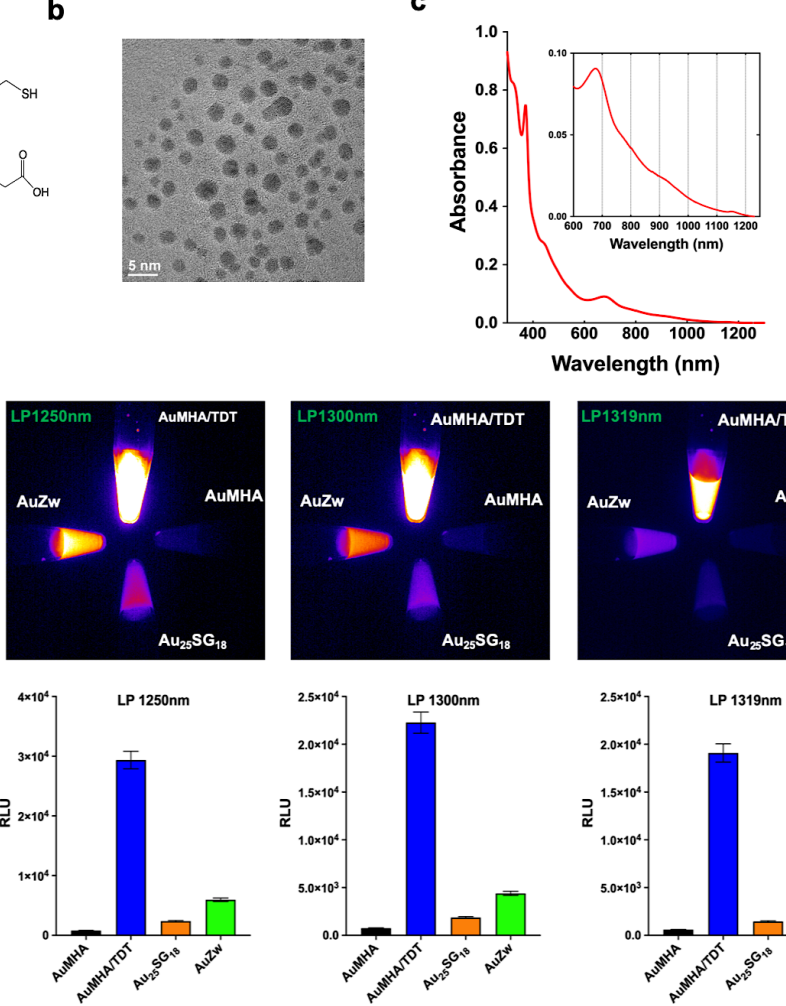
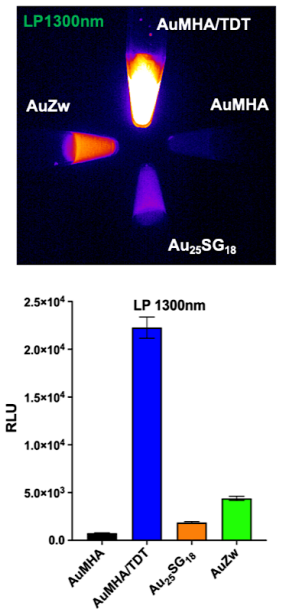
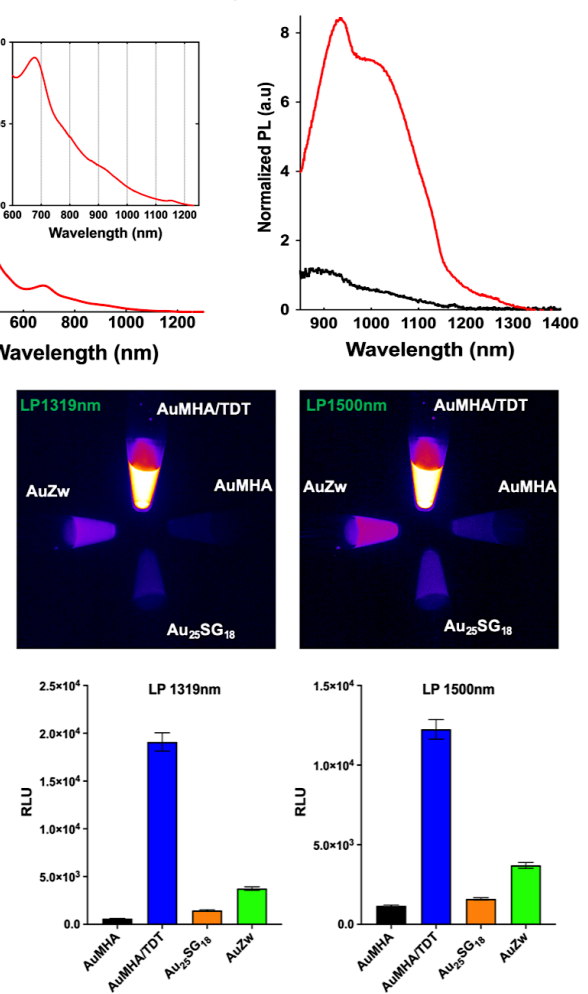
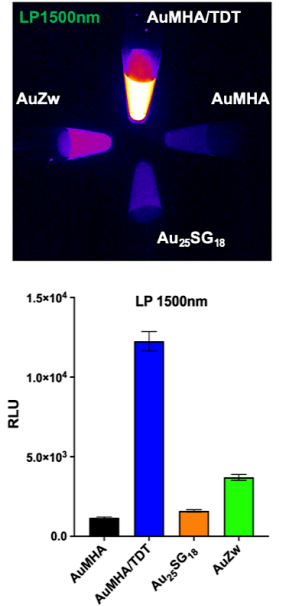

Figure 1.a. Scheme of the Au NCs AuMHA/TDT. b. HR-TEM images of AuMHA/TDT. c. Absorbance spectra of AuMHA/TDT. d. PL spectra of AuMHA (black line) and AuMHA/TDT (red line) $\left(\lambda_{\text {exc. }} 830 \mathrm{~nm}\right)$. e. SWIR PL of AuMHA, AuMHA/TDT, $\mathrm{Au}_{25} \mathrm{SG}_{18}$, and $A u Z w ~\left(300 \mu \mathrm{M}\right.$ in water) under NIR excitation ( $\left.\lambda_{\text {exc. }} 830 \mathrm{~nm}\right)$ using LP1064 nm $(1 \mathrm{~ms})$, LP1250 nm (5 ms), LP1300 nm (10 ms), LP1319 nm (25 ms), and LP1500 nm (250 ms). 
a

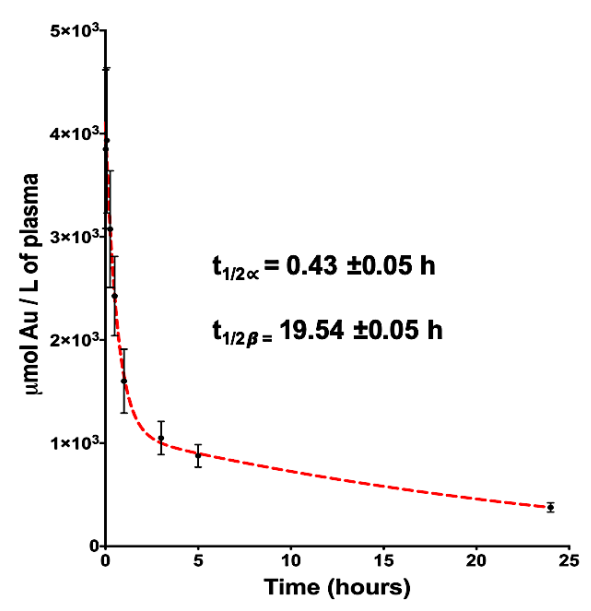

C

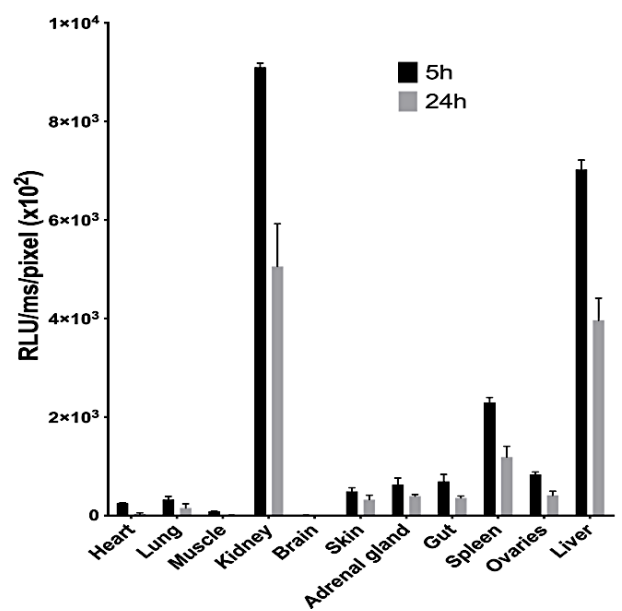

b

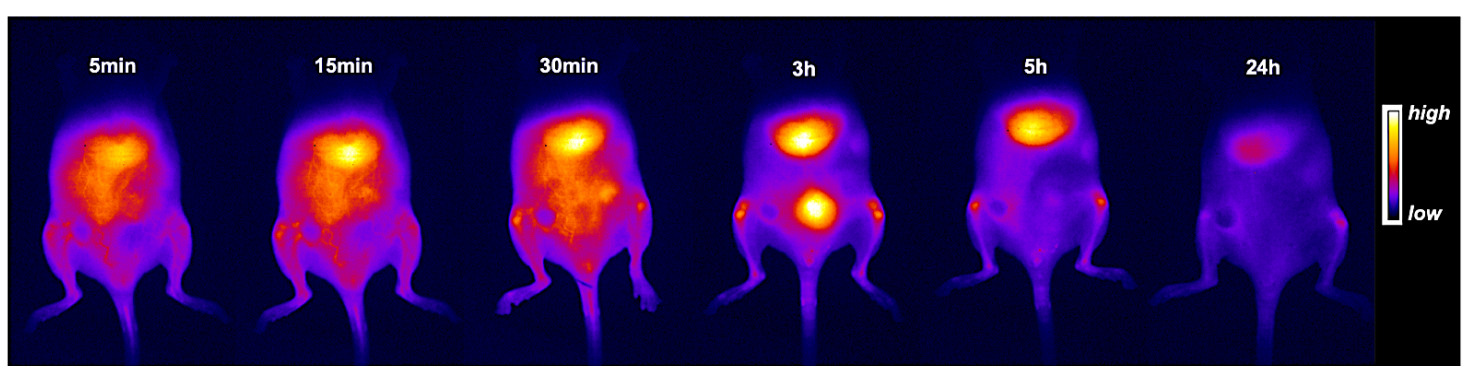

Figure 2. Biodistribution and pharmacokinetics of AuMHA/TDT after intravenous injection in nude mice ( $360 \mu \mathrm{M} ; 200 \mu \mathrm{L}$ per mouse).

a. In vivo pharmacokinetics determined by ICP-MS measurements on plasma samples taken at different time points ( $\mathrm{n}=4$ mice per time points). b. In vivo pharmacokinetic by fluorescence in mice over $24 \mathrm{~h}\left(\lambda_{\text {exc. }} 830 \mathrm{~nm}\right.$; LP1250 nm). c. Ex vivo 2D fluorescence signal of AuMHA/TDT in isolated organs $5 \mathrm{~h}$ and $24 \mathrm{~h}$ post injection ( $\mathrm{n}=3$ mice per time points). 

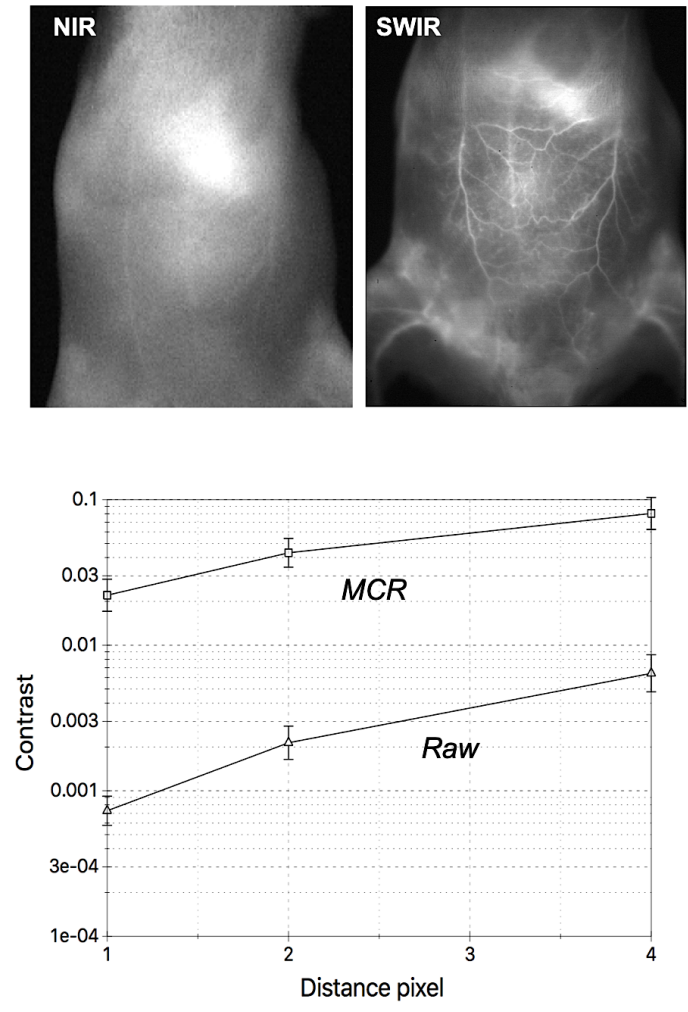
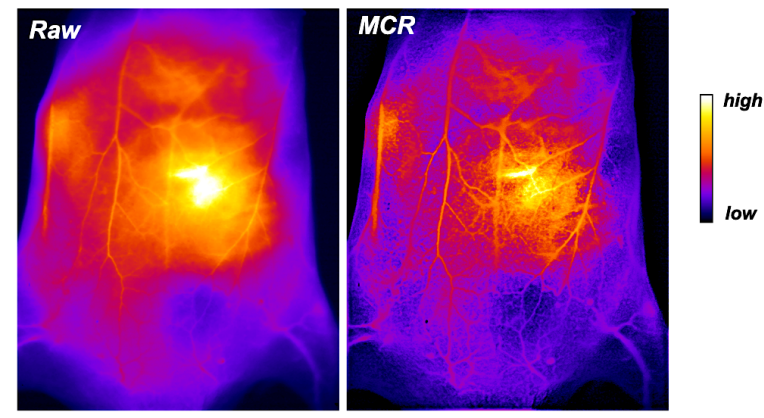

d
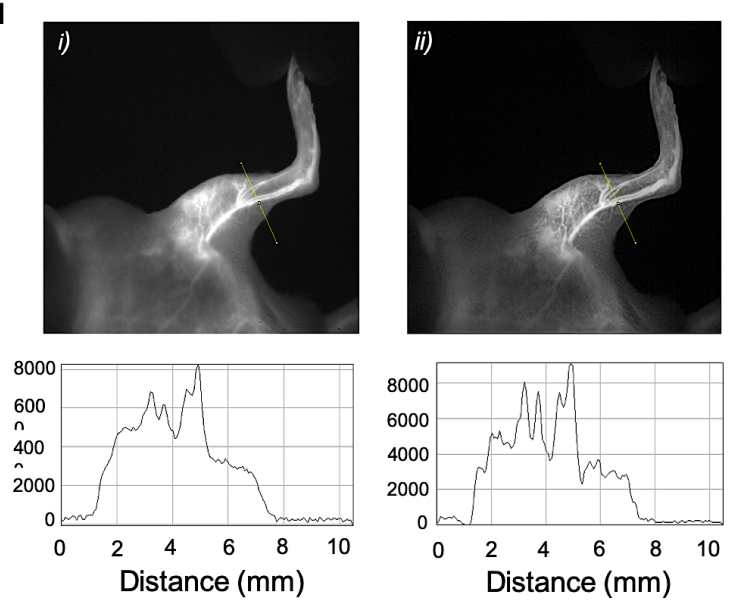

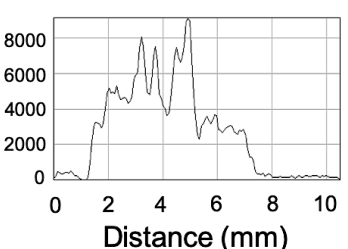

Figure 3. SWIR imaging of blood vasculature in WT 129/Ola mice after intravenous injection of AuMHA/TDT a. NIR I ( $\lambda_{\text {exc. }} 780 \mathrm{~nm} ; \lambda_{\text {em. }}>830 \mathrm{~nm}$ ) and (b) SWIR imaging (LP1250 nm) of mice 15 min after i.v injection of AuMHA/TDT $(200 \mu \mathrm{L}$ at $360 \mu \mathrm{M}$ in PBS). b. Noninvasive imaging of the mouse ventral area before (Raw) and after MCR processing (false colors). b. Quantification of contrast enhancement per pixel with and without MCR processing on the whole ventral area. c. Images of the left leg of the mouse (i) before and (ii) after MCR processing with their respective intensity profile across a line of interest drawn in the inset images above. 
a

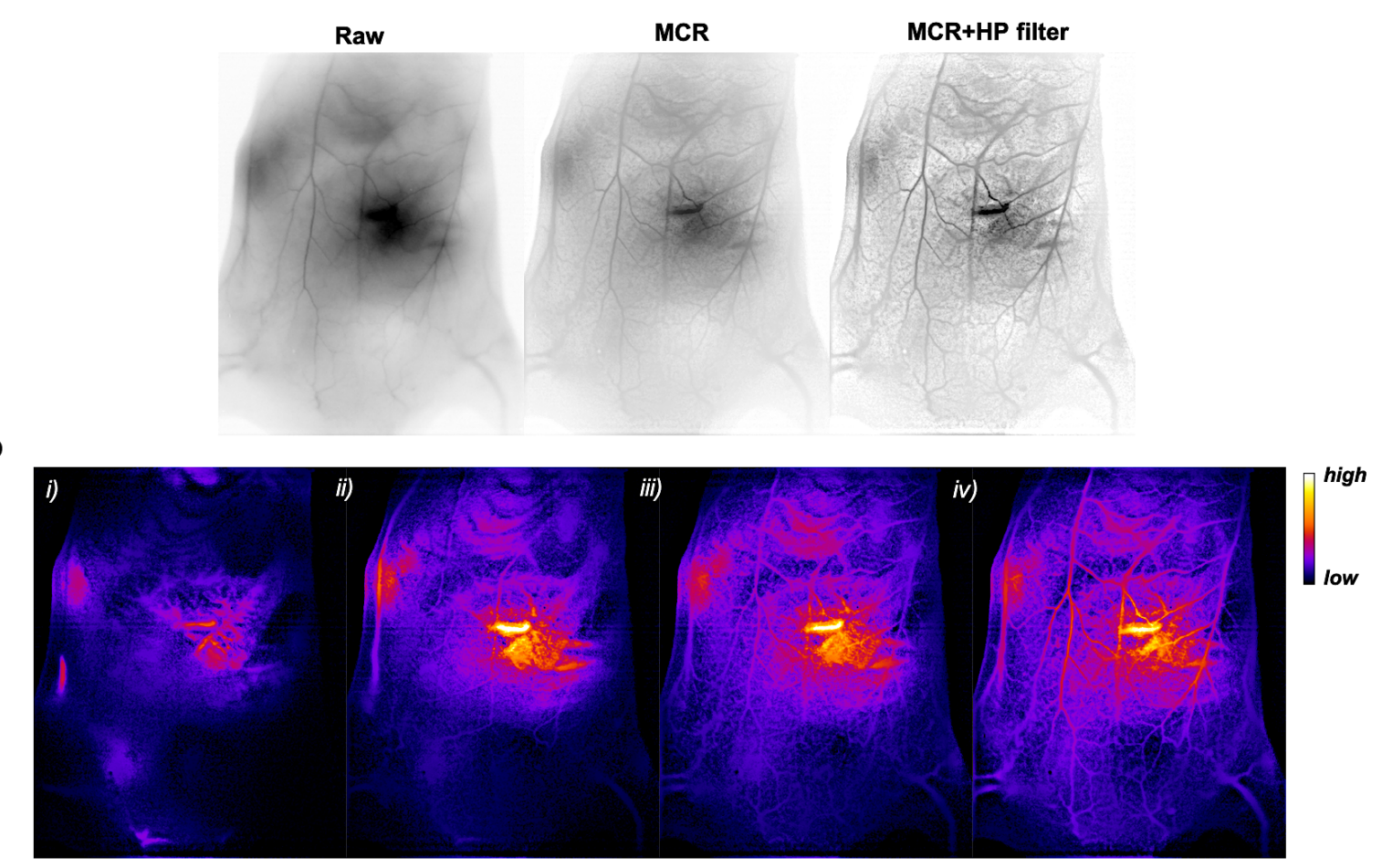

Figure 4. a. In vivo SWIR imaging (reverse contrast) of WT 129/Ola mice vasculature before imaging processing (raw) and after Monte Carlo constrained restoration (MCR) and an additional filtering (MCR+HP filter). b. MCR+HP filter treated SWIR images (false colors) i) $1.5 \mathrm{~s}$, ii) $5 \mathrm{~s}$, iii) $25 \mathrm{~s}$, and iv) $65 \mathrm{~s}$ after AuMHA/TDT bolus intravenous injection $(360 \mu \mathrm{M} ; 200$ $\mu \mathrm{L})$. 
a
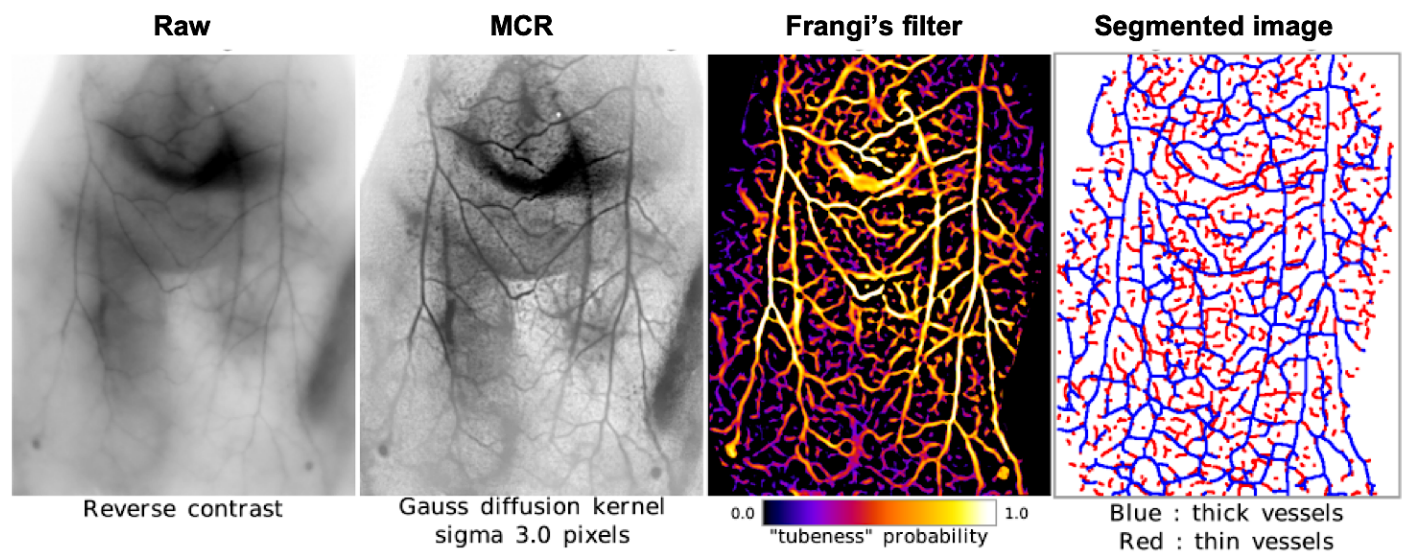

b
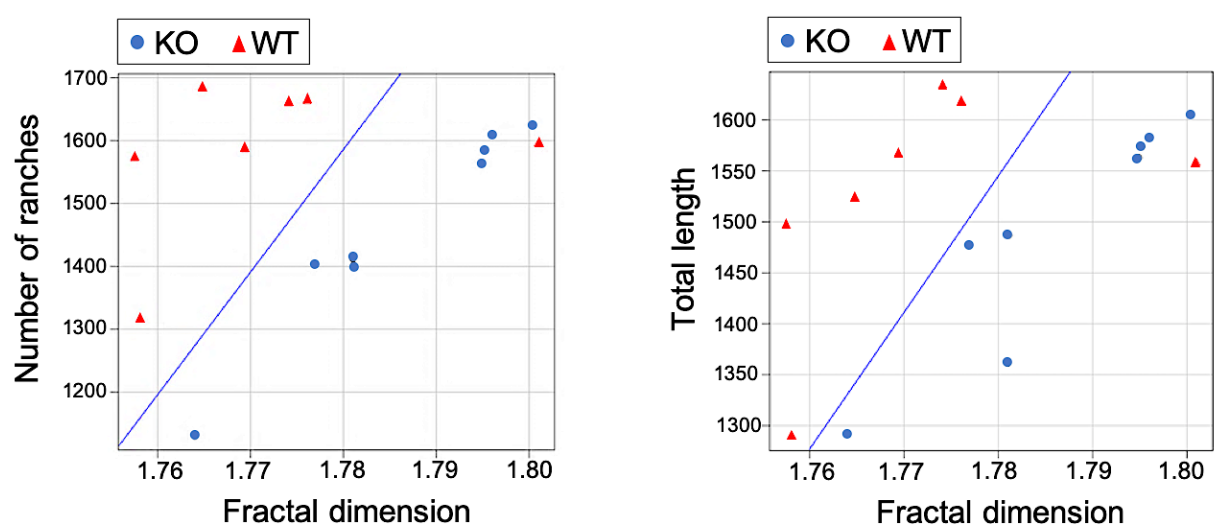

Figure 5.a. SWIR images of a Bmp $9-\mathrm{KO}$ mouse after MCR processing, Frangi's filter, and segmentation. b. Statistical analyses of blood vessel length and branches as a function of fraction dimension performed on Bmp9-KO mice $(\mathrm{n}=4)$ and WT mice $(\mathrm{n}=4)$. The blue line highlights the discrimination between the two groups. 
Ultra-small gold particles are used as SWIR contrast agent to detect a vascular disorder with a high level of precision non-invasively in depth and in real time using whole-body optical imaging.

Keyword: shortwave infrared fluorescence, gold nanoclusters, vascular disorder, monte carlo restoration imaging processing

Zhixi Yu ${ }^{1}$, Benjamin Musnier ${ }^{1}$, Maxime Henry ${ }^{1}$, K. David Wegner ${ }^{2}$, Benoit Chovelon $^{3}$, Agnès Desroche-Castan ${ }^{4}$, Ute Resch-Genger ${ }^{2}$, Sabine Bailly ${ }^{4}$, Jean-luc Coll $^{1^{*}}$, Yves Usson ${ }^{5}$, Véronique Josserand ${ }^{1}$, Xavier Le Guével ${ }^{1 *}$

High-resolution of Vascular Disorders by Shortwave Infrared Imaging using Gold Nanoclusters
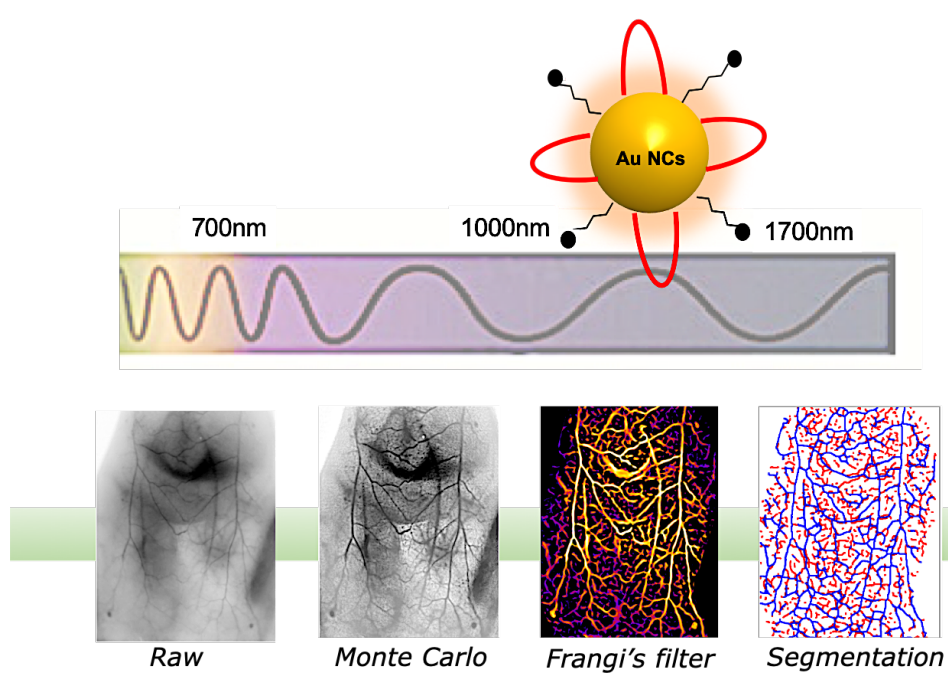


\section{REFERENCES}

1. Hong, G.; Antaris, A.; Dai, H., Near-infrared fluorophores for biomedical imaging. Nature biomedical engineering 2017, 1, 0010 (1-22).

2. Hong, G.; Diao, S.; Chang, J.; Antaris, A. L.; Chen, C.; Zhang, B.; Zhao, S.; Atochin, D. N.; Huang, P. L.; Andreasson, K. I.; Kuo, C. J.; Dai, H., Through-skull fluorescence imaging of the brain in a new near-infrared window. Nature Photonics 2014, 8 (9), 723-730. 3. Hong, G.; Lee, J. C.; Robinson, J. T.; Raaz, U.; Xie, L.; Huang, N. F.; Cooke, J. P.; Dai, H., Multifunctional in vivo vascular imaging using near-infrared II fluorescence. Nature Medicine 2012, 18 (12), 1841-1846.

4. $\quad$ Bruns, O.; Bischof, T.; Harris, D.; Franke, D.; Shi, Y.; Riedemann, L.; Bartelt, A.; Jaworski, F.; Carr, J.; Rowlands, C.; Wilson, M.; Chen, O.; Wei, H.; Hwang, G.; Montana, D.; Coropceanu, I.; Achorn, O.; Kloepper, J.; Heeren, J.; So, P.; Fukumura, D.; Jensen, K.; Jain, R.; Bawendi, M., Next-generation in vivo optical imaging with short-wave infrared quantum dots. Nature biomedical engineering 2017, 1, 0056(1-11).

5. $\quad$ Zhong, Y.; Ma, Z.; Wang, F.; Wang, X.; Yang, Y.; Liu, Y.; Zhao, X.; Li, J.; Du, H.; Zhang, M.; Cui, Q.; Zhu, S.; Sun, Q.; Wan, H.; Tian, Y.; Liu, Q.; Wang, W.; Garcia, K. C.; Dai, H., In vivo molecular imaging for immunotherapy using ultra-bright near-infrared-IIb rare-earth nanoparticles. Nature Biotechnology 2019, 37 (11), 1322-1331.

6. Yang, Q.; Ma, Z.; Wang, H.; Zhou, B.; Zhu, S.; Zhong, Y.; Wang, J.; Wan, H.; Antaris, A.; Ma, R.; Zhang, X.; Yang, J.; Zhang, X.; Sun, H.; Liu, W.; Liang, Y.; Dai, H., Rational Design of Molecular Fluorophores for Biological Imaging in the NIR-II Window. Advanced Materials 2017, 29 (12).

7. Porret, E.; Le Guével, X.; Coll, J.-L., Gold nanoclusters for biomedical applications: toward in vivo studies. Journal of Materials Chemistry B 2020.

8. Chen, Y.; Montana, D.; Wei, H.; Cordero, J.; Schneider, M.; Le Guevel, X.; Chen, O.; Bruns, O.; Bawendi, M., Shortwave Infrared in Vivo Imaging with Gold Nanoclusters. Nanoletters 2017, 17 (10), 6330-6334.

9. Desroches-Castan, A.; Tillet, E.; Ricard, N.; Ouarné, M.; Mallet, C.; Feige, J.-J.; Bailly, S., Differential Consequences of Bmp9 Deletion on Sinusoidal Endothelial Cell Differentiation and Liver Fibrosis in 129/Ola and C57BL/6 Mice. Cells 2019, 8 (9).

10. Desroches-Castan, A.; Tillet, E.; Ricard, N.; Ouarné, M.; Mallet, C.; Belmudes, L.; Couté, Y.; Boillot, O.; Scoazec, J.-Y.; Bailly, S.; Feige, J.-J., Bone Morphogenetic Protein 9 Is a Paracrine Factor Controlling Liver Sinusoidal Endothelial Cell Fenestration and Protecting Against Hepatic Fibrosis. Hepatology 2019, 70 (4), 1392-1408.

11. Musnier, B.; Wegner, K. D.; Comby-Zerbino, C.; Trouillet, V.; Jourdan, M.; Hausler, I.; Antoine, R.; Coll, J. L.; Resch-Genger, U.; Le Guevel, X., High photoluminescence of shortwave infrared-emitting anisotropic surface charged gold nanoclusters. Nanoscale 2019, 11 (25), 12092-12096.

12. Le Guevel, X.; Henry, M.; Motto-Ros, V.; Longo, E.; Montanez, M. I.; Pelascini, F.; de La Rochefoucauld, O.; Zeitoun, P.; Coll, J. L.; Josserand, V.; Sancey, L., Elemental and optical imaging evaluation of zwitterionic gold nanoclusters in glioblastoma mouse models. Nanoscale 2018, 10 (39), 18657-18664.

13. Shen, D.; Henry, M.; Trouillet, V.; Comby-Zerbino, C.; Bertorelle, F.; Sancey, L.; Antoine, R.; Coll, J. L.; Josserand, V.; Le Guével, X., Zwitterion functionalized gold nanoclusters for multimodal near infrared fluorescence and photoacoustic imaging. $A P L$ Materials 2017, 5 (5).

14. Liu, H.; Hong, G.; Luo, Z.; Chen, J.; Chang, J.; Gong, M.; He, H.; Yang, J.; Yuan, X.; Li, L.; Mu, X.; Wang, J.; Mi, W.; Luo, J.; Xie, J.; Zhang, X.-D., Atomic-Precision Gold Clusters for NIR-II Imaging. Advanced Materials 2019, 31 (46), 1901015. 
15. Carr, J. A.; Franke, D.; Caram, J. R.; Perkinson, C. F.; Saif, M.; Askoxylakis, V.; Datta, M.; Fukumura, D.; Jain, R. K.; Bawendi, M. G.; Bruns, O. T., Shortwave infrared fluorescence imaging with the clinically approved near-infrared dye indocyanine green. Proceedings of the National Academy of Sciences of the United States of America 2018, 115 (17), 4465-4470.

16. Du, B.; Jiang, X.; Das, A.; Zhou, Q.; Yu, M.; Jin, R.; Zheng, J., Glomerular barrier behaves as an atomically precise bandpass filter in a sub-nanometre regime. Nature Nanotechnology 2017, 12 (11), 1096-1102.

17. Tang, S.; Peng, C.; Xu, J.; Du, B.; Wang, Q.; Vinluan Iii, R. D.; Yu, M.; Kim, M. J.; Zheng, J., Tailoring Renal Clearance and Tumor Targeting of Ultrasmall Metal Nanoparticles with Particle Density. Angewandte Chemie International Edition 2016, 55 (52), 16039-16043. 18. Tillet, E.; Bailly, S., Emerging roles of BMP9 and BMP10 in hereditary hemorrhagic telangiectasia. Frontiers in Genetics 2015, 5, 456.

19. Frieden, B. R.; Zoltani, C. K., Monte Carlo restoration of binary objects. J. Opt. Soc. Am. A 1986, 3 (5), 731-734.

20. Colicchio B.; Xu C.; Haeberlé O.; Dieterlen A.; S. , J., Monte-carlo reconstruction of images from a psf variant system. Focus on Microscopy 2002. Technical Digest of Focus on Microscopy 2002, 56.

21. Frangi, A. F.; Niessen, W. J.; Vincken, K. L.; Viergever, M. A. In Multiscale vessel enhancement filtering, Medical Image Computing and Computer-Assisted Intervention MICCAI'98, Berlin, Heidelberg, 1998//; Wells, W. M.; Colchester, A.; Delp, S., Eds. Springer Berlin Heidelberg: Berlin, Heidelberg, 1998; pp 130-137.

22. Soleilhac, A.; Bertorelle, F.; Comby-Zerbino, C.; Chirot, F.; Calin, N.; Dugourd, P.; Antoine, R., Size Characterization of Glutathione-Protected Gold Nanoclusters in the Solid, Liquid and Gas Phases. The Journal of Physical Chemistry C 2017, 121 (49), 27733-27740. 